\title{
Lifetime of excited electrons in transition metals
}

\author{
Mário Rui Bacelar, ${ }^{1}$ Wolf-Dieter Schöne, ${ }^{1,2}$ Robert Keyling, ${ }^{1}$ and Walter Ekardt ${ }^{1}$ \\ ${ }^{1}$ Fritz-Haber-Institut der Max-Planck-Gesellschaft, Faradayweg 4-6, D-14195 Berlin, Germany \\ ${ }^{2}$ Freie Universität Berlin, Fakultät für Physik, Arnimallee 14, D-14195 Berlin, Germany
}

(Received 5 April 2002; published 4 October 2002)

\begin{abstract}
We present $a b$ initio calculations for the lifetime of excited electrons in transition metals. The computations were done using a pseudopotential approach in connection with a plane-wave expansion of the wave functions. The lifetimes for each element are resolved for various bands and with respect to certain directions of the crystal momentum. Our results reveal rather different trends for different transition metals showing the impossibility to work with simple models, thus emphasizing the need for first-principles calculations.
\end{abstract}

PACS number(s): 78.47.+p, 72.15.Lh, 71.10.-w, 71.20.Gj

Over the last few years the lifetime of excited electrons in metals has attracted considerable attention, both from the experimental $^{1-8}$ and the theoretical side. ${ }^{9-17}$ While experimental studies date back to the mid 1990's, the first theoretical calculations which really took into account the explicit band structure of the investigated systems were not published until 1999. ${ }^{9,10}$ Before this time the experimental data had to be compared to predictions based on the homogeneous electron gas (Fermi-liquid theory). ${ }^{18-20}$ Since then these so-called $a b$ initio calculations have been performed for simple metals ${ }^{9,12}$ and noble metals. ${ }^{9-13,16}$ Very recently results have been presented for the averaged lifetimes for bcc and fcc transition metals. ${ }^{17}$

These calculations clearly show that a treatment from first principles is extremely important in order to explain the experimental results. As an example we just would like to mention the case of the lifetime of excited electrons in $\mathrm{Al}$ for which an $a b$ initio calculation has been performed ${ }^{9}$ and the lifetime for the various excited states was calculated. This allowed a resolution with respect to the crystal momentum (wave vector) of the states. The calculation showed that the experiment ${ }^{5}$ - which was conducted on a polycrystalline sample_-did not probe the lifetime of excited electrons in the parabolic bands which dominate the band structure of $\mathrm{Al}$ but rather the lifetime of states which are not free-electron-like. In other words, Fermi-liquid theory fails to explain this experiment. Another example we would like to refer to is the case of $\mathrm{Cu}$. After reliable ab initio calculations ${ }^{9,10,12}$ were not able to explain the experimental data ${ }^{4}$ it became clear that measuring the lifetime of excited electrons using timeresolved two-photon photoemission experiments might lead under certain conditions to physical processes which cannot be explained just by electron-electron scattering and that other mechanisms ${ }^{21,22}$ may play an important role in these cases. This analysis was only made possible because trustworthy first-principle calculations had been available.

In this paper we present the calculations of the lifetime of excited electrons in six transition metals, two fcc metals (Rh and $\mathrm{Pd}$ ), two bcc metals ( $\mathrm{Nb}$ and $\mathrm{Mo}$ ), and two hcp metals (Y and $\mathrm{Ru}$ ). For all elements we present the lifetime resolved with respect to the crystal momentum of the corresponding states.

In this work we follow a procedure similar to the one already described in detail in previous work. ${ }^{9,11}$ We start by solving the Kohn-Sham (KS) equations ${ }^{23,24}$ of densityfunctional theory ${ }^{24,25}$ within the local-density approximation for the exchange-correlation potential. ${ }^{24}$ The wave functions are expanded with respect to a plane-wave basis set. ${ }^{26}$ The electron-ion interaction is described by means of a nonlocal, norm-conserving pseudopotential. ${ }^{27}$

After having obtained well-converged results for the ground state, the lifetimes of electrons in excited states is calculated using the so-called $G W$ approximation of manybody perturbation theory. ${ }^{28-31}$ In this approach it is assumed that the finite lifetimes of electronic excited states is due only to the scattering of the excited electron with electrons in the valence bands ("Fermi sea"). In the $G W$ approximation the electronic interaction is not described by the bare Coulomb potential but rather by a dynamically screened Coulomb potential. ${ }^{14}$ Within this formalism the lifetime is related to the imaginary part of the self-energy of the excited electron by $9,14,18-20$

$$
\begin{aligned}
\tau_{\mathbf{q}, j}^{-1} & =-\frac{2}{\hbar} \int d^{3} r d^{3} r^{\prime} \varphi_{\mathbf{q}, j}^{*}(\mathbf{r}) \operatorname{Im} \Sigma\left(\mathbf{r}, \mathbf{r}^{\prime} ; E_{\mathbf{q}, j}\right) \varphi_{\mathbf{q}, j}\left(\mathbf{r}^{\prime}\right) \\
& =-\frac{2}{\hbar} \operatorname{Im} \Sigma_{j=j^{\prime}}\left(\mathbf{q}, E_{\mathbf{q}, j}\right),
\end{aligned}
$$

where $\Sigma\left(\mathbf{r}, \mathbf{r}^{\prime} ; E_{\mathbf{q}, j}\right)$ is the self-energy of the excited electron and $\varphi_{\mathbf{q}, j}(\mathbf{r})$ and $E_{\mathbf{q}, j}$ are the wave function (in our case the $\mathrm{KS}$ wave function) and energy of the excited electron, respectively. $\mathbf{q}$ and $j$ denote a crystal momentum in the Brillouin zone and a band index. The second line in Eq. (1) is the definition of the representation of the self-energy with respect to the Bloch functions which are the solutions of the KS equations.

In Hedin's original work, ${ }^{28,29}$ the $G W$ approximation meant solving self-consistently a set of coupled integral equations retaining only the first order term of an expansion of the self-energy in terms of the screened Coulomb potential. Here, as in all previous work in which the $G W$ approximation is utilized in order predict or reproduce experimental data, ${ }^{30}$ we replace the full Green's function by the Green's function of noninteracting electrons and simply do not solve Hedin's original set of equations. We therefore obtain the self-energy within the random-phase approximation (RPA) ${ }^{32}$ 
and from this self-energy we determine the energy and lifetime of the excited electron state. So in practice the selfenergy is calculated as ${ }^{30,33,34}$

$$
\widetilde{\Sigma}\left(\mathbf{r}, \mathbf{r}^{\prime} ; t\right)=i G_{\mathrm{LDA}}\left(\mathbf{r}, \mathbf{r}^{\prime} ; t\right) W\left(\mathbf{r}, \mathbf{r}^{\prime} ; t^{+}\right)-v_{\mathrm{xc}}(\mathbf{r}) \delta\left(\mathbf{r}-\mathbf{r}^{\prime}\right)
$$

Here $v_{\mathrm{xc}}(\mathbf{r})$ is the exchange-correlation potential as used in the KS equations, $G_{\mathrm{LDA}}\left(\mathbf{r}, \mathbf{r}^{\prime} ; t\right)$ is the Green's function of the excited KS electron and $W\left(\mathbf{r}, \mathbf{r}^{\prime} ; t\right)$ is the shielded potential calculated within the RPA. The self-energy is determined in the basis of the solutions of the KS equations ${ }^{9}$ [Bloch basis, see second line in Eq. (1)]. Since it has been shown that in crystalline systems the off-diagonal elements of the self-energy can be neglected ${ }^{34,35}$ we only calculate the diagonal elements. The energy of the excited electron $E_{\mathbf{q}, j}$ is determined by solving ${ }^{9,32}$

$$
E_{\mathbf{q}, j}-\epsilon_{\mathbf{q}, j}-\operatorname{Re} \widetilde{\Sigma}_{j=j^{\prime}}\left(\mathbf{q}, E_{\mathbf{q}, j}\right)=0,
$$

where $\epsilon_{\mathbf{q}, j}$ are the (real) eigenvalues of the KS equations.

In a recent article $^{36}$ it was shown that in order to obtain a reliable occupied band width and $d$-band energies which are comparable to the experimental data for $\mathrm{Cu}$ it is necessary to consider the $4 s$ and $4 p$ semicore states as valence states when constructing the pseudopotential which is used for the ground-state calculation. However, in almost all calculations of the lifetime of excited electrons pseudopotentials have been utilized in which the conventional choice for the valence electrons was used, namely, the $3 d$ and the $4 s$ electrons. ${ }^{9-14}$ The exceptions are two very recent determinations of the lifetime which are based on a full all-electron calculation. ${ }^{16,17}$ The results of all these calculations agree well with the experimentally available data. In the following we would like to shed some light on this ostensible discrepancy.

In their paper Marini et al. ${ }^{36}$ explain the influence of the semicore states, especially on the width and the energetic position of the $d$ bands in $\mathrm{Cu}$, with the huge change of the Fock part of the self-energy. As can be seen from Fig. 1 in Ref. 36, the inclusion of the semicore states leads to a decrease of the Fock part of the self-energy of up to $6 \mathrm{eV}$ for states with single-particle energies within the region of the $d$ bands. For the unoccupied states the Fock part of the selfenergy remains largely unaffected with respect to an inclusion of the semicore states. So there is a non-negligible contribution to the exchange part of the self-energy, which affects the calculated values of the electronic energies. It does not, however, affect the imaginary parts of the selfenergies, where the effects of the semicore states can be neglected. This is demonstrated at the example of three states at the $\Gamma$ point of the noble metal Ag and the transition metals $Y$ and Rh. For Ag and Rh the self-energy shown was calculated for occupied states within the $d$ bands. The change of the real part of the self-energy once the semicore states are considered can be nicely noted. The self-energy shown for $\mathrm{Y}$ was calculated for an unoccupied state. Here, too, the imaginary parts are nearly identical. This discussion shows that an in-
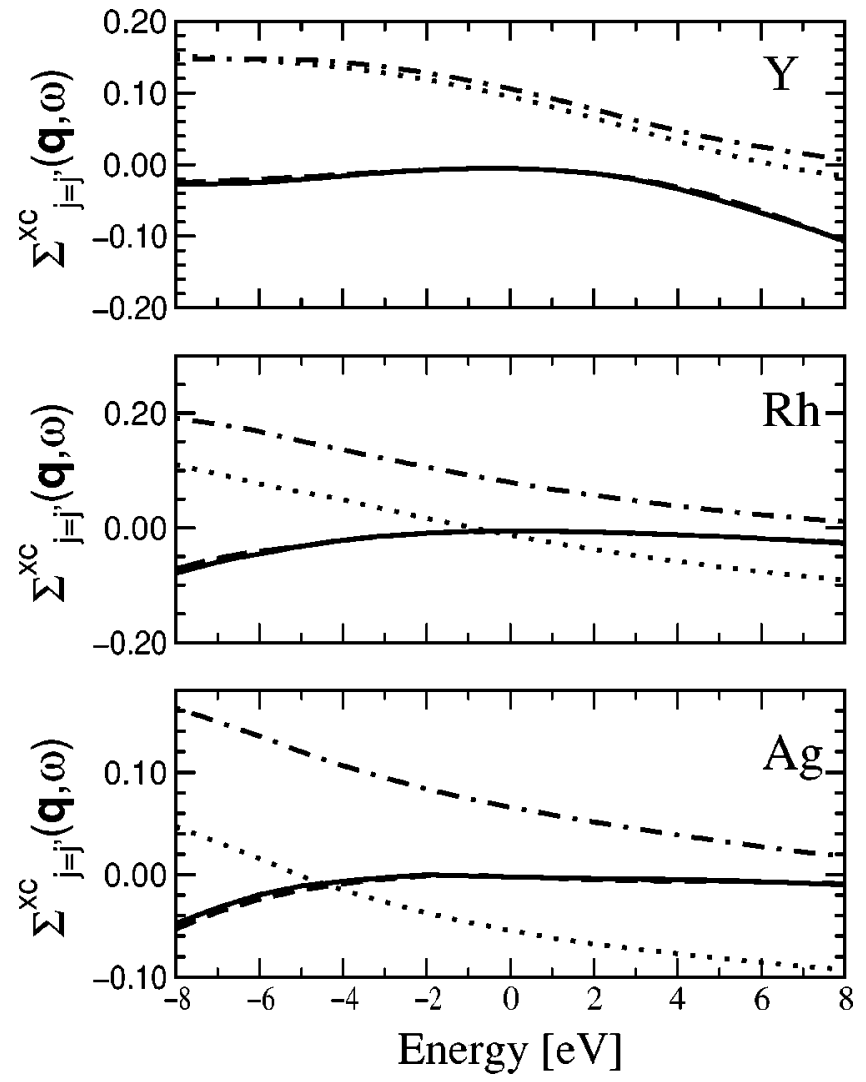

FIG. 1. Imaginary and real part of the self-energy at the $\Gamma$ point for Y (upper panel), Rh (middle panel), and Ag (bottom panel), calculated using only the $4 d$ and $5 s$ electrons as valence electrons (solid line for the imaginary parts, dot-dashed lines for the real parts) and considering also the $4 s$ and $4 p$ electrons as valence electrons (dashed line for imaginary parts, dot lines for real parts). For $\mathrm{Ag}$ and $\mathrm{Rh}$ the plots show the self-energies for occupied states within the $d$ bands (band numbers 5 and 4, respectively). For Y the self-energy of an unoccupied state was plotted (band number 4). All plots show only minor differences in the imaginary parts of the self-energy, whereas the real parts for $\mathrm{Ag}$ and $\mathrm{Rh}$ are markedly different. See main text for more details.

clusion of the semicore states is required only if the (quasiparticle) energies of occupied states within the $d$ bands are considered.

In Figs. 2-5 we present the calculated lifetimes for excited electrons for six transition metals in selected bands. In all cases the lifetime according to Eq. (1) was calculated in the so-called "energy-shell" approximation, i.e., we evaluated the self-energy not at the quasiparticle energy but at the KS energy value. ${ }^{10,12,13}$ Figure 2 shows the lifetime for electrons in the sixth band of $\mathrm{Rh}$ (solid line) and Pd (dashed line) for the directions $\Gamma-K$ (left panel) and $\Gamma-X$ (right panel). (Here and in the following the numeration of the bands was made with respect to increasing energy at the $\Gamma$ point.) With the exception of the energy range below $1 \mathrm{eV}$ in the $\Gamma-X$ direction the lifetime in $\mathrm{Rh}$ is smaller than in $\mathrm{Pd}$. This is in agreement with phase-space arguments; for a fixed energy the band structure of $\mathrm{Rh}$ offers more available empty states for the decay of excited electrons than the one of Pd. Note, however, that the overall lifetime for the two directions is 

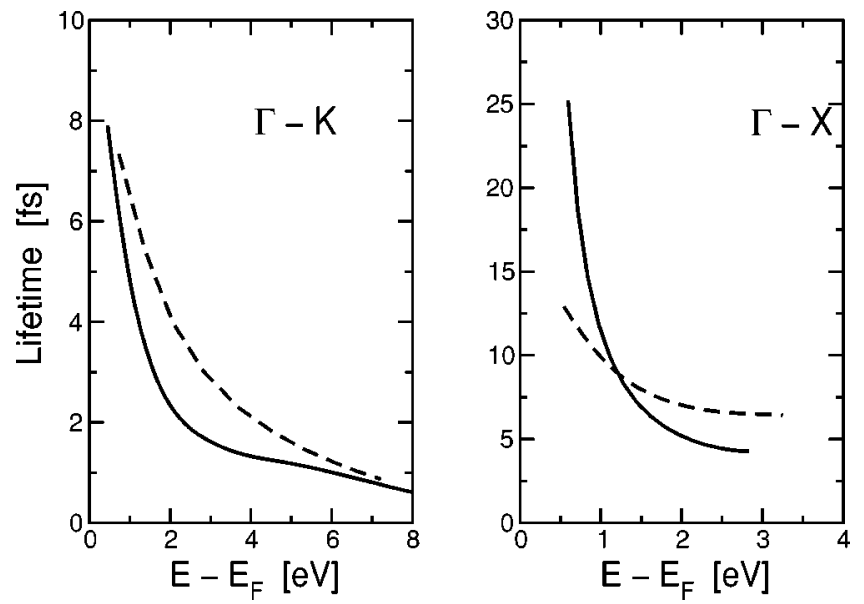

FIG. 2. Lifetime for the sixth band in the two fcc metals Rh (solid line) and $\mathrm{Pd}$ (dashed line) for the $\Gamma-K$ and $\Gamma-X$ directions. We labeled the bands with respect to increasing energy using the $\Gamma$ points as reference.

quite different which shows once more the need for crystal momentum resolved calculations.

Figure 3 shows the lifetime of excited electronic states in Mo (left panel) and $\mathrm{Nb}$ (right panel) for the bands 3, 4, 5, and 6 in the direction $\Gamma-K$. We find no relevant difference between the lifetimes for the various bands; the underlying band structure is identical for all bands. Apparently the explicit form of the bands has only a minor impact on the lifetimes as has the possibility for potential interband transitions. As in the previous case of Rh and Pd there is a clear difference between the lifetime of the two elements. As already noticed in Ref. 17, this cannot be explained by phasespace arguments which means that this line of reasoning has to be used with care. The lack of simple physical arguments or sum rules which might be used to predict or analyze the overall results for obtained lifetimes make reliable $a b$ initio calculations even more important.

It is instructive to compare our results with the data for the averaged lifetimes reported in Ref. 17. To the point we
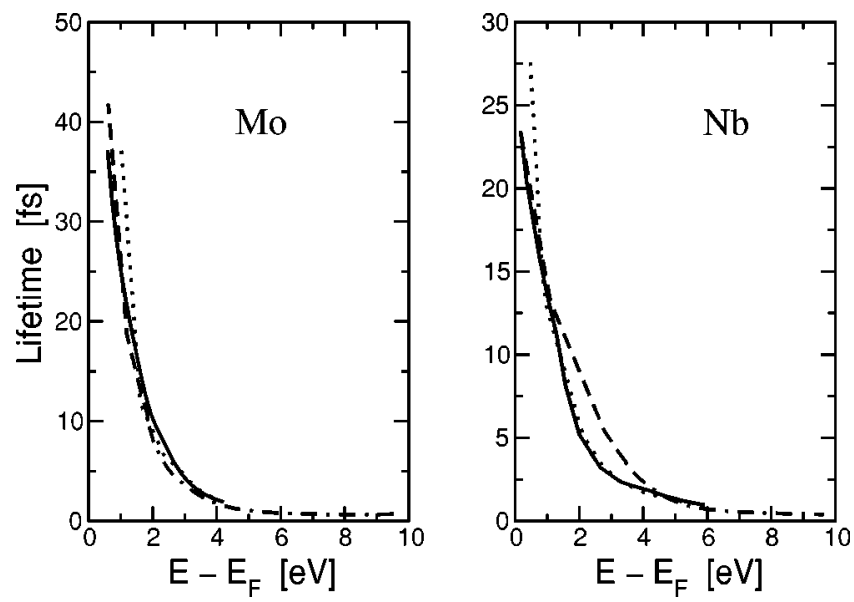

FIG. 3. Lifetime for bands 3 (solid line), 4 (dotted line), 5 (dashed line), and 6 (dot-dashed line) in the $\Gamma-K$ direction of the two bcc metals Mo and $\mathrm{Nb}$.
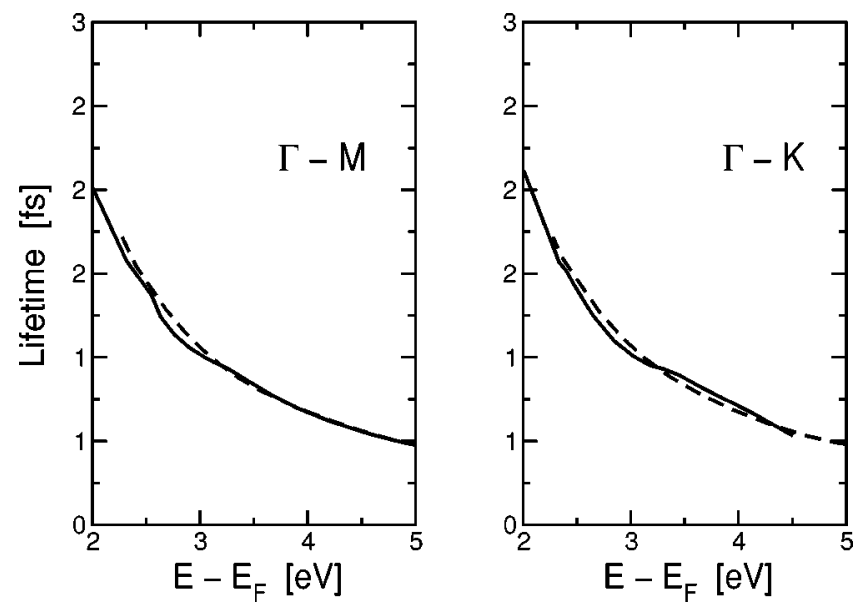

FIG. 4. Lifetime of electrons in excited states for the bands 6 (solid line) and 8 (dashed line) in $\mathrm{Y}$ in the $\Gamma-M$ and $\Gamma-K$ direction.

can compare our crystal momentum resolved results with the averaged lifetimes of Ref. 17 there is qualitative agreement. This is noteworthy because the two calculations differ in three points. First, we perform a pseudopotential calculation whereas in Ref. 17 all-electron calculations are reported. Second and connected to this are the different sets of basis functions, namely plane waves and a set of numerical muffin tin orbitals in the calculation of Zhukov et al. According to the arguments given in the paragraphs following Eq. (3) neither of the two points should have a significant impact on the results for the lifetimes. For the noble metals this has recently been shown explicitly. ${ }^{16}$ This provides a further justification for our approach of using only the $4 d$ and $5 s$ electrons as valence electrons in the pseudopotential approach. The third difference between the two calculations concerns the energy at which the imaginary part of the self-energy is evaluated in order to obtain the lifetime. Whereas in this work the energy-shell approximation is utilized ${ }^{10,12,13}$ Zhukov and co-workers evaluate the self-energy at the quasiparticle energy. In other words, their results for the lifetime should be slightly larger, the results differing by the renor-
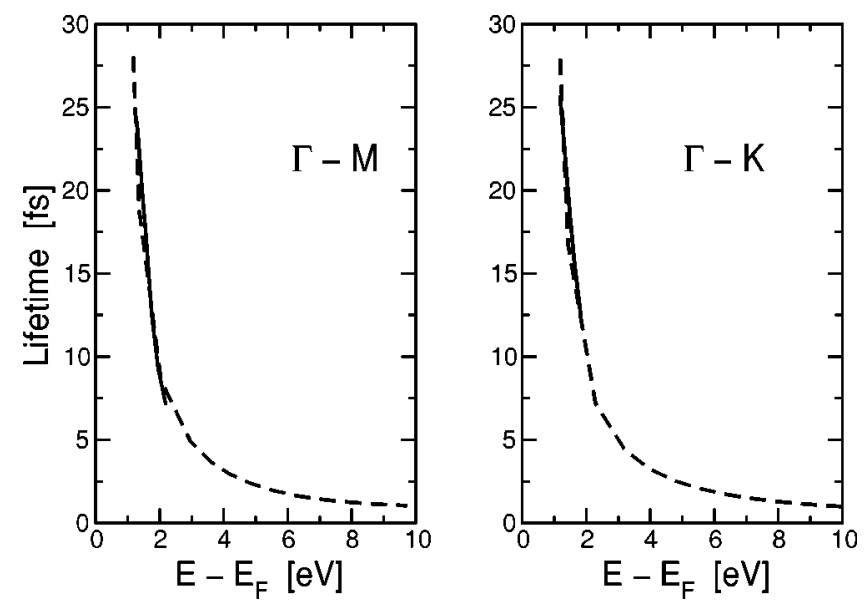

FIG. 5. Lifetime of electrons in excited states for the bands 11 (solid line) and 12 (dashed line) in Ru in the $\Gamma-M$ and $\Gamma-K$ directions. 
malization constant $Z_{k} \cdot{ }^{9}$ This is indeed the general trend which can be seen. An exception is the lifetimes of excited electrons in the $\Gamma-X$ direction in $\mathrm{Rh}$ and $\mathrm{Pd}$ which are slightly larger than the averaged lifetimes reported in Ref. 17.

Figure 4 shows the lifetimes of electrons in excited states in $\mathrm{Y}$ for the bands 6 (solid line) and 8 (dashed line) for the directions $\Gamma-M$ (left panel) and $\Gamma-K$ (right panel). As in the previous cases there is a negligible difference in the lifetimes for bands in the same direction, and contrary to the fcc metals considered in this work, the results for the $\Gamma-M$ and $\Gamma-K$ directions are very similar.

Finally, Fig. 5 shows the lifetimes of electrons in excited states in $\mathrm{Ru}$ for the bands 11 (solid line) and 12 (dashed line) in the same directions as for Y. Again we predict for the same direction, lifetimes relatively independent of the band for which they are calculated. And as in the $\mathrm{Y}$ case there is no relevant difference for the two directions considered.

In summary we have presented calculations for the lifetime of excited electrons in transition metals. The fact that in transition metals neither the simple phase-space argument nor other easy to apply rules of thumb can be used to predict the lifetime of excited electrons confirms the need for elaborated $a b$ initio calculations for these kinds of systems. Our results also emphasize the need for crystal momentum resolved calculations.

G. Ertl is gratefully acknowledged for his interest and generous support. This work was supported by the Deutsche Forschungsgemeinschaft through SFB 450.
${ }^{1}$ M. Aeschlimann, M. Bauer, and S. Pawlik, Chem. Phys. 205, 127 (1996).

${ }^{2}$ S. Pawlik, M. Bauer, and M. Aeschlimann, Surf. Sci. 377-379, 206 (1997).

${ }^{3}$ H. Petek and S. Ogawa, Prog. surf. Sci. 56, 239 (1997).

${ }^{4}$ S. Ogawa, H. Nagano, and H. Petek, Phys. Rev. B 55, 10869 (1997).

${ }^{5}$ M. Bauer, S. Pawlik, and M. Aeschlimann, Proc. SPIE 3272, 201 (1998).

${ }^{6}$ E. Knoesel, A. Hotzel, and M. Wolf, Phys. Rev. B 57, 12812 (1998).

${ }^{7}$ J. Cao, Y. Gao, H.E. Elsayed-Ali, R.J.D. Miller, and D.A. Mantell, Phys. Rev. B 58, 10948 (1998).

${ }^{8}$ M. Wolf, A. Hotzel, E. Knoesel, and D. Velic, Phys. Rev. B 59, 5926 (1999).

${ }^{9}$ W.-D. Schöne, R. Keyling, M. Bandić, and W. Ekardt, Phys. Rev. B 60, 8616 (1999).

${ }^{10}$ I. Campillo, J.M. Pitarke, A. Rubio, E. Zarate, and P.M. Echenique, Phys. Rev. Lett. 83, 2230 (1999).

${ }^{11}$ R. Keyling, W.-D. Schöne, and W. Ekardt, Phys. Rev. B 61, 1670 (2000).

${ }^{12}$ I. Campillo, V.M. Silkin, J.M. Pitarke, E.V. Chulkov, A. Rubio, and P.M. Echenique, Phys. Rev. B 61, 13484 (2000).

${ }^{13}$ I. Campillo, J.M. Pitarke, A. Rubio, and P.M. Echenique, Phys. Rev. B 62, 1500 (2000).

${ }^{14}$ P.M. Echenique, J.M. Pitarke, E.V. Chulkov, and A. Rubio, Chem. Phys. 251, 1 (2000).

${ }^{15}$ W. Ekardt, W.-D. Schöne, and R. Keyling, Appl. Phys. A: Mater. Sci. Process. 71, 529 (2000).

${ }^{16}$ V.P. Zhukov, F. Aryasetiawan, E.V. Chulkov, I.G. de Gurtubay, and P.M. Echenique, Phys. Rev. B 64, 195122 (2001).
${ }^{17}$ V.P. Zhukov, F. Aryasetiawan, E.V. Chulkov, and P.M. Echenique, Phys. Rev. B 65, 115116 (2002).

${ }^{18}$ J.J. Quinn and R.A. Ferrell, Phys. Rev. 112, 812 (1958).

${ }^{19}$ R.H. Ritchie, Phys. Rev. 114, 644 (1959).

${ }^{20}$ J.J. Quinn, Phys. Rev. 126, 1453 (1962).

${ }^{21}$ W.-D. Schöne and W. Ekardt, Phys. Rev. B 62, 13464 (2000).

${ }^{22}$ W.-D. Schöne and W. Ekardt, Phys. Rev. B 65, 113112 (2002).

${ }^{23}$ W. Kohn and L.J. Sham, Phys. Rev. 137, A1697 (1965).

${ }^{24}$ R.M. Dreizler and E.K.U. Gross, Density Functional Theory (Springer, Berlin, 1990).

${ }^{25}$ P. Hohenberg and W. Kohn, Phys. Rev. 136, B864 (1964).

${ }^{26}$ M. Bockstedte, A. Kley, J. Neugebauer, and M. Scheffler, Comput. Phys. Commun. 107, 187 (1997).

${ }^{27}$ N. Troullier and J.L. Martins, Phys. Rev. B 43, 1993 (1991).

${ }^{28}$ L. Hedin, Phys. Rev. 139, A796 (1965).

${ }^{29}$ L. Hedin and S. Lundqvist, in Solid State Physics: Advances in Research and Applications, edited by H. Ehrenreich, F. Seitz, and D. Turnbull (Academic Press, New York, 1969), Vol. 23, p. 1.

${ }^{30}$ F. Aryasetiawan and O. Gunnarsson, Rep. Prog. Phys. 61, 237 (1998).

${ }^{31}$ W. G. Aulbur, L. Jönsson, and J. W. Wilkins, in Solid State Physics: Advances in Research and Applications, edited by H. Ehrenreich and F. Spaepen (Academic Press, New York, 1999), Vol. 54 , p. 1.

${ }^{32}$ G. D. Mahan, Many-Particle Physics (Plenum, New York, 1990).

${ }^{33}$ W.-D. Schöne and A.G. Eguiluz, Phys. Rev. Lett. 81, 1662 (1998); 83, 241 (1999); 83, 242 (1999).

${ }^{34}$ M.S. Hybertsen and S.G. Louie, Phys. Rev. B 34, 5390 (1986).

${ }^{35}$ L. Hedin, Int. J. Quantum Chem. 56, 445 (1995).

${ }^{36}$ A. Marini, G. Onida, and R. Del Sole, Phys. Rev. Lett. 88, 016403 (2002). 